\title{
Microbiota metabolite short chain fatty acids, GPCR, and inflammatory bowel diseases
}

\author{
Mingming Sun ${ }^{1,2} \cdot$ Wei Wu ${ }^{1,2} \cdot$ Zhanju Liu $^{2} \cdot$ Yingzi Cong ${ }^{1,3}$
}

Received: 12 July 2016/Accepted: 12 July 2016/Published online: 23 July 2016

(C) Japanese Society of Gastroenterology 2016

\begin{abstract}
Gut microbiota has been well recognized in regulation of intestinal homeostasis and pathogenesis of inflammatory bowel diseases. However, the mechanisms involved are still not completely understood. Further, the components of the microbiota which are critically responsible for such effects are also largely unknown. Accumulating evidence suggests that, in addition to pathogenassociated molecular patterns, nutrition and bacterial metabolites might greatly impact the immune response in the gut and beyond. Short chain fatty acids (SCFA), which are metabolized by gut bacteria from otherwise indigestible fiber-rich diets, have been shown to ameliorate diseases in animal models of inflammatory bowel diseases (IBD) and allergic asthma. Although the exact mechanisms for the action of SCFA are still not completely clear, most notable among the SCFA targets is the mammalian G protein-coupled receptor pair of GPR41 and GPR43. In addition to the well-documented inhibition of histone deacetylases activity mainly by butyrate and propionate, which causes anti-inflammatory activities on IEC, macrophages, and dendritic cells, SCFA has recently been implicated in promoting development of Treg cells and possibly other $\mathrm{T}$ cells. In addition to animal models, the beneficial effects have also
\end{abstract}

M. Sun and W. Wu contributed equally.

Yingzi Cong

yicong@utmb.edu

1 Department of Microbiology and Immunology, University of Texas Medical Branch, 4.142C Medical Research Building, 301 University Blvd, Galveston, TX 77555-1019, USA

2 Department of Gastroenterology, The Shanghai Tenth People's Hospital, Tongji University, Shanghai, China

3 Department of Pathology, University of Texas Medical Branch, Galveston, TX, USA been reported from the clinical studies that used SCFA therapeutically in controlled trial settings in inflammatory disease, in that application of SCFA improved indices of IBD and therapeutic efficacy was demonstrated in acute radiation proctitis. In this review article, we will summarize recent progresses of SCFA in regulation of intestinal homeostasis as well as in pathogenesis of IBD.

Keywords Microbiota - Short chain fatty acids - GPCR . IBD

\section{Introduction}

The crucial role of gut microbiota has been well-established in regulation of the intestinal homeostasis and inflammatory bowel diseases (IBD). However, the components of the microbiota which are critically responsible for such effects are still largely unknown. Emerging evidence suggests that the host immune system can sense gut bacterial metabolites in addition to pathogen-associated molecular patterns (PAMP) and that recognition of these small molecules can influence the host immune response in the context of disease and inflammation in the gut and beyond [1-3]. Of particular interest are short chain fatty acids (SCFA), such as acetate, $n$-propionate, and $n$-butyrate, which are solely metabolized by gut bacteria from otherwise indigestible carbohydrates, i.e., from fiber-rich diets [4], and have been shown to ameliorate disease in animal models of colitis and allergic asthma [5, 6]. Furthermore, SCFA are associated with reduced risk of various diseases, including IBD, and dysbiosis in IBD patients has been associated with altered SCFA fermentative pathways $[6,7]$. In this review, we will focus on SCFA regulation of host immune responses and pathogenesis of IBD. 


\section{Formation of SCFA}

Mammalian gastrointestinal (GI) tract harbors huge amounts of diverse microbes, comprising more than 1000 strains [8]. This commensal microbiota not only contributes to the regulation of host immune response and homeostasis, but also participates in the breakdown of food and energy metabolism [9, 10]. Gut bacteria have enzymes that host cells lack for breaking down carbohydrates, turning them into different useful metabolites. In recent years, microbiota-derived metabolites, including SCFA, phenolic acids, tryptophan, and bile acids, have drawn greater attention $[11,12]$. Usually undigested dietary fibers, as well as proteins and peptides, can be fermented in the cecum and colon by gut bacteria. The major products of these fermentative reactions are SCFA, which are defined as the groups of fatty acids with fewer than six carbons, including formic acid (C1), acetic acid (C2), propionic acid (C3), butyric acid (C4) and valeric acid (C5). The major SCFA in gut are $\mathrm{C} 2, \mathrm{C} 3$ and $\mathrm{C} 4$, which account for more than $95 \%$ of all the SCFA. In general, C2 can be formed from pyruvate via acetyl-CoA or Wood-Ljungdahl pathway [13]. C3 is mainly produced from succinate via the succinate pathway or from lactate via the acrylate pathway. C4 is formed from acetyl-CoA and butyryl-CoA, as well as acetate and lactate [14]. The concentration of SCFA varies from cecum to colon. It is estimated that the total amount in the proximal colon ranges from 70 to $140 \mathrm{mM}$ and falls to $20-70 \mathrm{mM}$ in the distal colon. After being absorbed, SCFA are utilized in the colonocytes or transported to blood circulation and other organs. In general, SCFA enter cells through several different ways, the first one being passive diffusion, the second being carriermediated transportation by SMCT1/Slc5a8 and MCT1/S1$\mathrm{c} 16 \mathrm{a} 1$, and the third being activating G-protein-coupled receptors (GPCR). Slc5a8, which is a $\mathrm{Na}^{+}$-coupled highaffinity transporter especially for butyrate, has been shown to protect against colitis and colon cancer under low-fiber dietary conditions. It regulates butyrate-induced expression of IDO1 and Aldh1A2 in dendritic cells (DCs) as well as development of Treg cells [15, 16]. Slc16a1 transports SCFA depending on the net chemical gradients for $\mathrm{H}^{+}$ [17]. GPR41, GPR43 and GPR109a are the receptors that could be activated by SCFA (Fig. 1). SCFA-GPR pathways
Fig. 1 Formation, absorption and transportation of SCFA. Indigestible dietary fibers can be fermented in the cecum and colon by gut microbes to form SCFA, which are usually utilized in the enterocytes through different ways: passive diffusion; carrier-mediated transportation by Slc5a 8 and Slc16a1; and binding GPR41, GPR43 and GPR109a. After being absorbed, SCFAs are transported into portal vein via superior mesenteric vein and inferior mesenteric vein depending on the absorption sites, and dispersed to peripheral tissues such as skeletal muscle, liver and adipose tissue to take effects
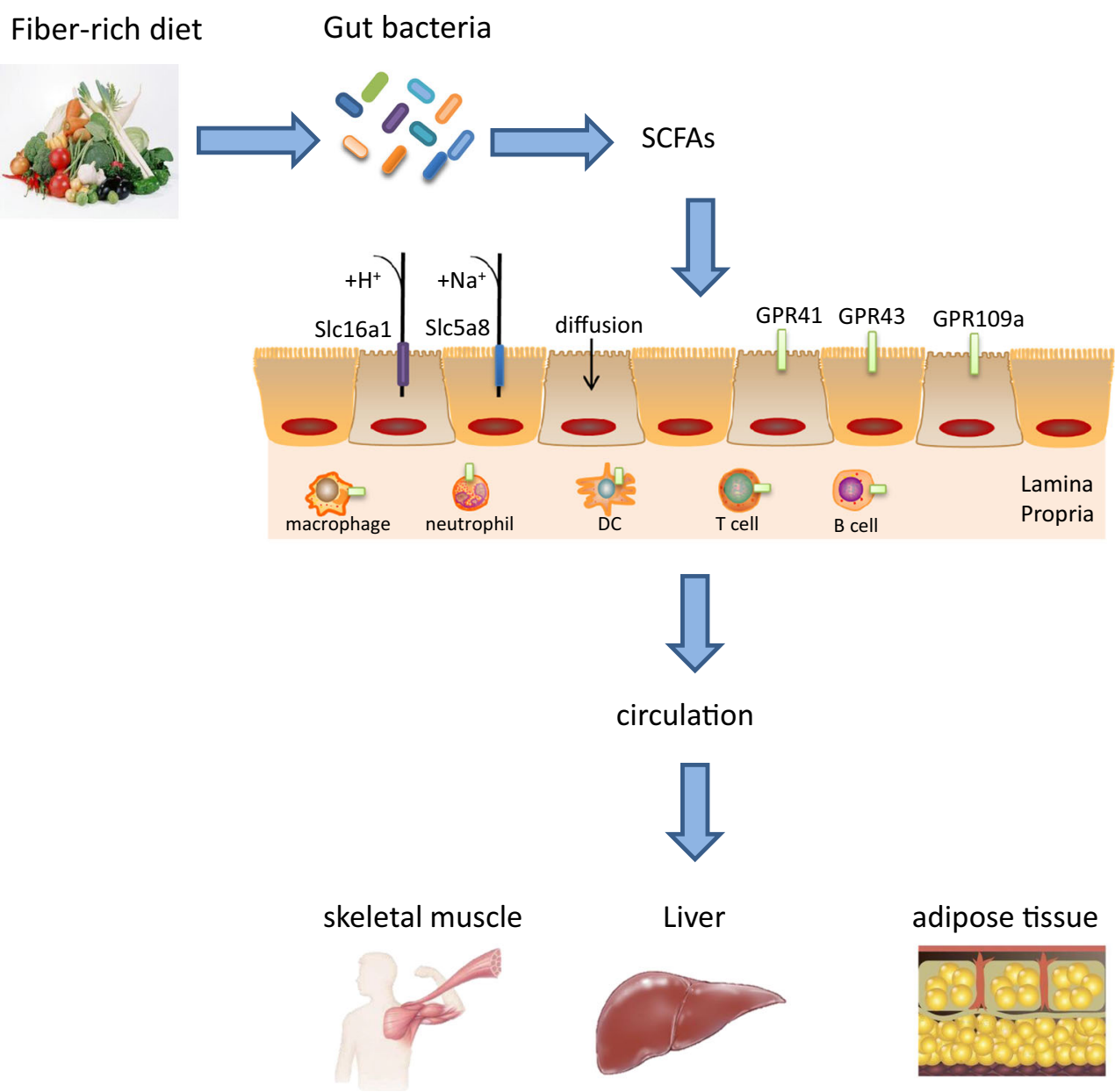
have been demonstrated to play vital roles in regulation of immune responses [18, 19].

\section{SCFA in gut and circulation}

SCFA are the most abundant products derived from commensal bacterial fermentation of indigestible dietary fibers in intestines [14]. The principal SCFA in the gut are acetate, propionate and butyrate, which constitute more than $95 \%$ of all the SCFA content [4]. Their concentrations in the gut are typically found in a ratio of 3:1:1 [20]. Interestingly, different SCFA vary in different sites throughout the whole intestines: acetate and propionate are found in both small and large intestines, while butyrate is found mainly in the colon and cecum [21]. It has been shown that about 400-800 mmol SCFA are produced with a high-fiber diet per day, equaling that $10 \mathrm{~g}$ of dietary fiber fermentation. Multiple factors, including diet, specific diversity of gut microbiota and certain amount of commensal bacteria, play a vital role in the production of SCFA [22]. After production, SCFA can be absorbed into colonic epithelial cells through several ways: non-ionic diffusion; via the carrier-mediated transportation; and exchange with bicarbonate [23]. Moreover, SCFA that are absorbed in the cecum, ascending colon and transverse colon enter the superior mesenteric vein, while SCFA that are absorbed in the descending colon and sigmoid are transported into the inferior mesenteric vein. Then both of them drain into the portal vein and liver [24]. Apart from this pathway, SCFA absorbed in the rectum could drain into the inferior vena cava through the pelvic plexus and play roles in the circulation. After entering circulation, SCFA have been demonstrated to affect metabolism and the function of peripheral tissues such as modulating adipose tissue, skeletal muscle and liver as well (Fig. 1) [22].

\section{Gut SCFA-producing bacteria}

According to the formation of three main SCFA, acetate can be produced from pyruvate by two different ways: via acetyl-CoA by enteric bacteria and via Wood-Ljungdahl by acetogens; for instance, Blautia hydrogenotrophica. Butyrate is produced from Acetyl-CoA by several Firmicutes. Propionate is produced by two different pathways: the succinate pathway by Bacteroidetes and the lactate pathway by Firmicutes [11]. The microbial conversions of undigested fibers to SCFA are mediated by specific members of gut bacteria. Recent advances in technology using pyrosequencing analyses of $16 \mathrm{~S}$ rRNA genes have made great progress in characterization of bacteria responsible for SCFA production. Propionate and butyrate production pathways appear more conserved and substrate specific, while pathways for acetate production are widely distributed among bacterial groups. Although distributed across a number of phyla, propionate production is dominated by relatively few bacterial genera [25]. Deoxy-sugars, such as fucose and rhamnose, are particularly propiogenic in select organisms [25]. A small number of gut bacteria, including Faecalibacterium prausnitzii, Eubacterium rectale, Eubacterium hallii and Ruminococcus bromii, are responsible for the majority of butyrate production [26]. Fermentation of resistant starch greatly contributes to butyrate production in the colon, which is dominated by Ruminococcus bromii [27].

\section{SCFA regulation of immune response}

Gut microbiota was recently found to participate in regulation of several systems of the body through their metabolites, principally SCFA produced in colon and also absorbed in blood circulation to reach other organs. Therefore, SCFA modulate functions of different systems, such as gut, nervous, endocrine and blood, serving as a key factor to regulate metabolic disorders and immunity, primarily through the inhibition of histone deacetylases and the activation of G-protein coupled receptors such as GPR41, GPR43 and GPR109a [28]. The effects of SCFA have been demonstrated in influencing systemic autoimmune responses and participating in different steps of inflammation process. SCFA are found to regulate the functions of almost every type of immune cell, altering gene expression, differentiation, chemotaxis, proliferation and apoptosis (Fig. 2).

During innate immune responses at mucosal sites, microbial products are recognized by pattern-recognition receptors, such as toll-like receptors. SCFA affect pro-inflammatory cytokines production (e.g., IL-6, IL-8, IL-1 $\beta$ and TNF $\alpha$ ) through enhancing NF- $\kappa B$ activation in TLR ligand-responses in epithelial cells [29]. During inflammation, SCFA stimulate the migration of neutrophils by activating GPR43 [30] and modulate their production of reactive oxygen species and phagocytosis [31]. Furthermore, SCFA could also inhibit pro-inflammatory cytokine production such as TNFa in neutrophils [32]. SCFA regulate the functions of DCs, which regulate immune response depending not only on secretion of cytokines, but also on their ability to interact with $\mathrm{T}$ cells. Butyrate and propionate inhibit activation of BMDC via suppressing the LPS-induced expression of co-stimulatory molecule CD40 and secretion of IL-6 and IL-12p40 [33]. A recent study demonstrated that DCs exposed to butyrate could facilitate the differentiation of naïve $\mathrm{T}$ cells into $\mathrm{FoxP}^{+}{ }^{+}$regulatory T-cells (Tregs), and inhibit the differentiation of naïve $\mathrm{T}$ cells into interferon (IFN)- $\gamma$-producing cells through butyrate induced expression of immunosuppressive enzymes indoleamine 2,3-dioxygenase 1 (IDO1) and 


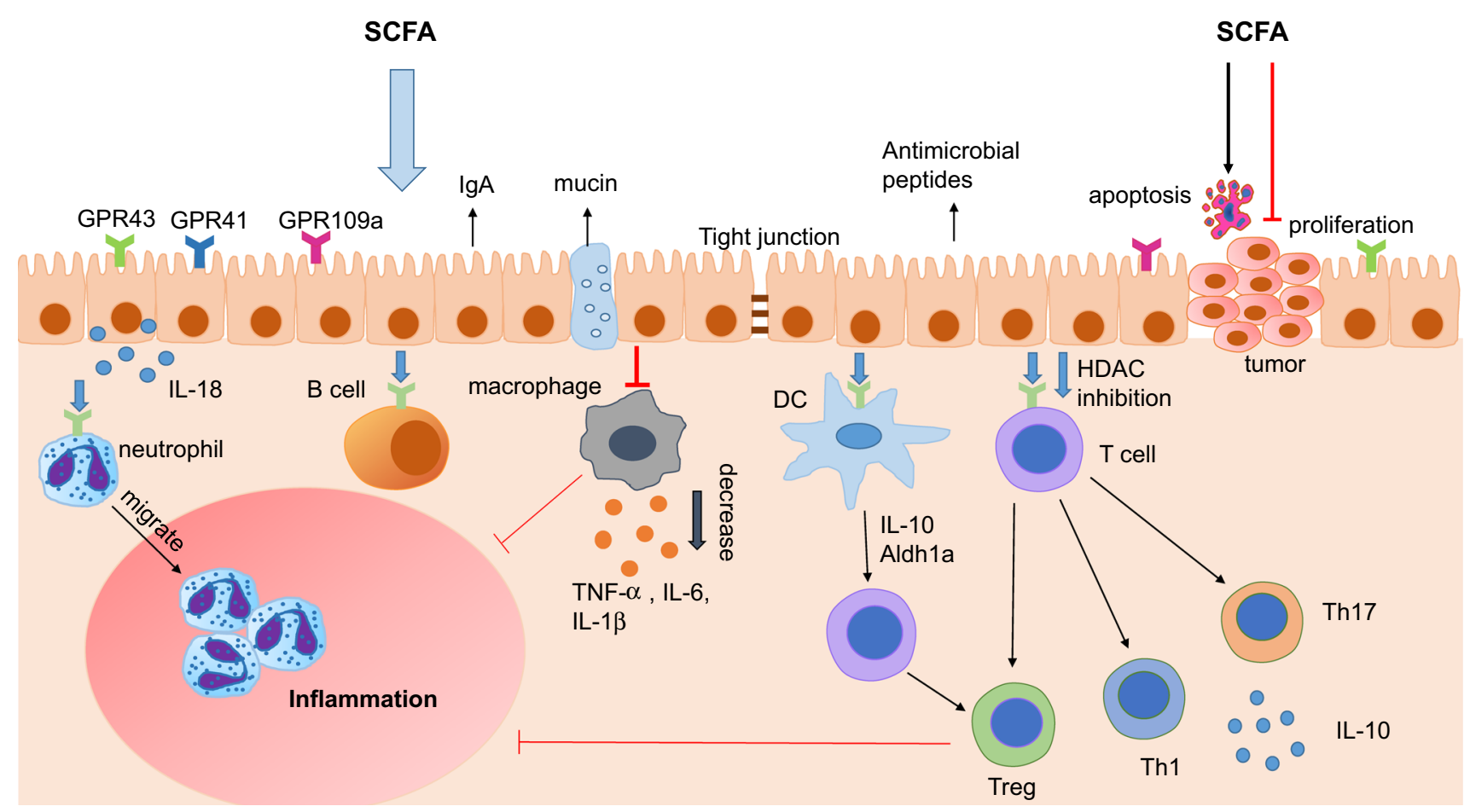

Fig. 2 SCFA regulation of intestinal immunity. SCFA regulate the intestinal mucosal immunity through exerting their effects on various immune cells. SCFA regulate intestinal barrier integrity by inducing intestinal epithelial cell secretion of IL-18, antimicrobial peptides, mucin, and upregulating the expression of tight junction. SCFA induce neutrophils migration to inflammatory site and enhance their phagocytosis. SCFA regulate the $\mathrm{T}$ cell function not only through the protein-coupled receptor (GPCR) pathway but also inhibition of

aldehyde dehydrogenase 1A2 (Aldh1A2) [15]. Furthermore, DCs from mice treated with propionate, characterized by reduced expression of CD40, PD-L2 and CD86, exhibited the impaired ability to initiate Th2 effector function and further to promote Th2-mediated allergic airway inflammation [5]. Butyrate has also been shown to modulate the function of intestinal macrophages. Treatment of macrophages with butyrate inhibits LPS-induced proinflammatory mediators, including nitric oxide (NO), IL-6, and IL-12, but does not affect production of TNF- $\alpha$ or MCP-1. Interestingly, these effects are independent of TLR signaling and activation of GPRs, but dependent on the inhibition of HDAC by butyrate [34].

SCFA also regulate adaptive immune responses. Mice provided with SCFA had an increased number of extrathymic Foxp $3^{+}$Treg cells [35] and SCFA promoted conversion of naïve $\mathrm{T}$ cells toward Treg under Treg-cell polarization condition [36]. Butyrate upregulated the histone $\mathrm{H} 3$ acetylation of Foxp3 and promoted the differentiation of Treg, which acts as a key anti-inflammatory effector [35]. These data suggest that SCFA might influence histone acetylation whose transcription of genes is histone deacetylase (HDAC). The differentiation of $\mathrm{T}$ cells is mediated both by SCFA regulation of DCs and the direct act of SCFA on T cells. SCFA regulate the generation of Th1, Th17 and Treg in different cytokine milieus. SCFA also inhibit intestinal macrophage production of proinflamamtory cytokines through inhibition of HDAC, and possibly induce intestinal IgA production of $\mathrm{B}$ cells. Moreover, SCFAs inhibit the carcinogenesis through promoting apoptosis and suppressing proliferation of tumor cells

involved in differentiation of $\mathrm{T}$ cells. SCFA also enhance Foxp3 expression of colonic $\mathrm{T}$ cells via activation of GPR43 on T cells [3]. A recent study further reported that SCFA increase the IL-10 production in T cell, including Th1, Th17 and Treg cells [37]. However, it should be noted that, in this study, SCFA were also found to directly facilitate the conversion of naïve T cells into Th1 or Th17 depending on cytokine milieu. This effect of SCFA is primarily dependent on HDAC inhibitor activity, but independent of GPR43 [37]. It has also been shown that dietary cycloinulooligosaccharides increased SCFA and IgA production in mice, indirectly indicating that SCFA might promote the secretion of IgA by B cells [38].

\section{GPCR in SCFA regulation of immune responses}

SCFA-sensing G-protein-coupled receptors (GPCR), which are also called free fatty acid receptors (FFARs), include GPR41 (FFAR3), GPR43 (FFAR2) and GPR109 (hydroxycarboxylic acid receptor 2 or HCA2). GPR41 and GPR43 can be efficiently activated by acetate, propionate, butyrate and other SCFAs [39], while GPR109a can be activated 
mainly by butyrate and niacin [40, 41]. GPR41, GPR43 and GPR109a are coupled with $\mathrm{G}_{\mathrm{i} / 0}$ and their activation by SCFA inhibits cAMP production [42]. SCFA could regulate the immune responses through activating GPCR, which is expressed on almost all the immune cells, such as epithelial cells, neutrophils and macrophages [39]. Early works on GPCR revealed that GPCR-deficient mice exhibit severe and unrecovered inflammation in models of DSSinduced colitis, arthritis and asthma [43]. GPR41 signaling is essential to the protection of propionate in allergic airway inflammation (AAI). Propionate-treated GPR41 $1^{-1-}$ mice after house dust mite exposure did not exhibit the amelioration of AAI compared with pretreated WT and GPR $43^{-1-}$ mice [5]. Dimethyl fumarate (DMF) was found to protect against multiple sclerosis (MS) through its metabolites, which active GPR109a. DMF could reduce neutrophil infiltration to improve EAE, a mouse model of MS, in WT mice, but not GPR109a ${ }^{-1-}$ mice, suggesting a critical role of GPR109a in suppression of MS [44]. Following these discoveries, other studies demonstrated that GPR43 could regulate immune responses in a myriad of different ways. GPR43 on intestinal epithelial cells activates the NLRP3 inflammasome and enhances the production of IL-18, which is critical for maintaining epithelial integrity and intestinal homeostasis [19]. Recent studies showed that activation of the GPR43 pathway protected against colon cancer through inducing cancer apoptosis and inhibiting cancer cell proliferation [45]. GPR43 on neutrophils promotes polymorphonuclear leukocyte (PMN) recruitment to the inflammatory site, probably in a protein kinase $\mathrm{p} 38 \alpha$-dependent manner [30]. A protection of GPR $43^{-1-}$ mice from inflammatory tissue destruction in chronic DSS colitis was reported by diminished intestinal migration of PMN [46]. In the monosodium urate monohydrate (MSU)-induced gout model, GPR $43^{-/-}$ mice showed decreased neutrophil recruitment resulted from lower production of IL- $1 \beta$ in the course of inflammatory response [47]. Furthermore, acetate could inhibit LPS-induced TNF $\alpha$ secretion from both mice and human PBMCs by GPR43 pathway [48]. GPR43 on colonic T cells induces the differentiation and enhances the suppressive function of Foxp $3^{+}$Tregs through epigenetic modifications [3]. Taken together, these data suggest that SCFA-sensing GPCRs play an important role in regulation of immunity and inflammation.

\section{SCFA and GPCR regulation of IBD}

The mammalian gastrointestinal tract harbors trillions of bacteria. The metabolites of bacteria such as SCFA reach a high concentration in the gut. The gut is the primary site where SCFA mediate their effect on either intestinal epithelial integrity or mucosal immune responses. The disorder of gut microbiota leading to decreased SCFA is associated with colonic diseases, including IBD. In the often-quoted study, the fecal microbiota in European children who are susceptible to develop IBD showed lower bacterial richness and an absence of bacteria efficient at fiber digestion and SCFA production compared with African children [49]. Moreover, western diet caused microbiome perturbation, SCFA reduction and high risk of colitis. Particularly in this study, expression of GPR43 was found to decrease in CD patients as well as mice fed with a high fat and high sugar diet [50]. There is considerable evidence that the concentration of SCFA was decreased in the colonic lumen of ulcerative colitis (UC) [51]. Furthermore, a recent study suggested that the dybiosis, characterized by a decrease of the butyrate-producing species Roseburia hominis and Eaecalibacterium prausnitizii, was defined in patients with UC [7].

SCFA-sensing GPCR protect against the intestinal inflammation not only through intestinal epithelial barrier maintenance, but also immune regulation. Both GPR43 and GPR109a are important for regulation of gut immunity. It was found that GPR $43^{-/-}$and GPR $109 \mathrm{a}^{-/-}$mice suffered from more severe DSS-induced colitis [43, 52]. Several groups have found that SCFA could promote the generation and suppressive function of colonic Treg in antibiotic treated mice or GF mice. GPR43 on colonic $\mathrm{T}$ cells potentially induce the Treg differentiation when binding SCFA. Importantly, propionate or SCFA mix-treated $\mathrm{Rag}^{-1-}$ mice injected with naïve $\mathrm{T}$ cells and Treg had lower level of colitis than mice received water [3]. Butyrate binding to GPR109a endows the colonic DCs and macrophages with the ability to induce Treg generation by increasing their production of IL-10 and Aldh1a [52]. The intestinal epithelial barrier plays a critical role in preventing intestinal inflammation of IBD. SCFA affect intestinal epithelial cells that highly expressed GPR43, functioning as regulators of the physical barrier and secretion of mucin, antimicrobial peptides, chemokines and cytokines. SCFA might activate NALP6 through the GPCR pathway, further promoting gut goblet cells to secret mucus, which is an important barrier to separate the bacteria and epithelial cells $[53,54]$. Several recent studies also suggested that butyrate could upregulate the tight junction and regulate epithelial permeability [55]. Chronic hypoxia in intestinal mucosa of IBD results in several alterations of the gut environment. Stabilization of hypoxia inducible factors (HIFs) has been revealed to protect against inflammation and maintain intestinal homeostasis [56, 57]. A recent study has shown that SCFA were highly produced in low $\mathrm{O}_{2}$ condition and then regulated $\mathrm{O}_{2}$ metabolism in intestinal epithelial cells, and further maintained epithelial integrity and protected the barrier via increase HIF stabilization [58]. In contrast, GPR $43^{-/-}$and GPR $41^{-/-}$mice 
were found to have reduced colonic inflammation after administration of ethanol and 6-trinitrobenzene sulfonicacid (TNBS); or infection with Citrobacter rodentium, possibly resulting from decreased secretion of proinflammatory cytokines and chemokines in intestinal epithelial cells by promotion of SCFA [18]. Overall, SCFA are involved in both anti- and pro-inflammatory processes of IBD.

Chronic intestinal inflammation such as IBD increases the risk of colorectal carcinogenesis, which is called colitis-associated cancer. Gut microbiota is associated with the process of inflammation and tumorigenesis. Thus, their metabolites SCFA were observed to confer protection in the development of colon cancer, partially in the GPCRdependent manner. Moreover, the expression of GPR43 is reduced in human colon cancer. Propionate and butyrate induce cancer apoptosis and inhibit cancer cell proliferation, depending on GPR43 pathway [45]. GPR109a is the receptor for butyrate in colon, and is expressed on colonic epithelial cells and immune cells. GPR109a deficiency has been reported to promote inflammation-induced colon carcinogenesis. In addition, GPR $109 \mathrm{a}^{-1-}$ mice suffered from exacerbated colitis and carcinogenesis compared to WT mice treated with AOM + DSS, being associated with decreased production of IL-10 and IL-18 and increased production of IL-17 [52].

The beneficial effects of SCFA have also been reported in patients with IBD. It was proposed a long time ago to treat patients with IBD by administration of SCFA or prebiotics that are known to enhance SCFA production [59-62]. Treatment of SCFA on UC patients has been demonstrated to be effective to ameliorate colitis [63]. SCFA mixture (sodium acetate, sodium propionate and sodium butyrate) enemas, serving as an adjuvant therapy, enhanced the efficacy of classic IBD treatments such as 5-aminosalicylic acid and corticosteroid therapy [61]. Collectively, SCFA have profound effects on the regulation of gut immunity and the pathogenesis of IBD. However, due to partial restricted indications or patient compliance, these treatments have not been established as a standard of care.

\section{Conclusions}

The significance of interactions between dietary intake and gut microbiota has been well recognized in human health. The discovery that SCFA are the natural ligands for GPR41, GPR43, and GPR109a, which are expressed on a wide range of cell types, has led to re-emerged interest in the role of SCFA in human health, especially in regulation of inflammatory bowel diseases. With the accumulating data indicating regulatory function of SCFA in a wide range of immune cells, including both innate and adaptive cells, they represent a new frontier in manipulation and prevention of IBD. However, many questions remain to be investigated. For examples, how diets with high-fiber regulate gut microbiota composition and function, and what the role of SCFA is in such a process. While most studies are currently focusing on SCFA regulation of innate immune responses, there is the question of how they regulate gut adaptive immune responses, i.e., do they affect gut B cell development and antibody responses to microbiota as well as to food antigens? Or do they regulate gut $\mathrm{T}$ cell responses in addition to promote Treg cells? Can SCFA through high-fiber diets prevent as well as treat intestinal inflammation in chronic animal models and eventually in patients with IBD? Or do they function differently in patients with CD and UC? Understanding such questions will surely provide great insights into development of new therapeutic avenues to treating IBD patients through dietary manipulation.

Acknowledgments This work was supported by NIH Grants DK098370 and DK105585.

\section{Compliance with ethical standards}

Conflict of interest The authors declare that they have no conflict of interest.

\section{References}

1. Haghikia A, Jorg S, Duscha A, Berg J, Manzel A, Waschbisch A, et al. Dietary fatty acids directly impact central nervous system autoimmunity via the small intestine. Immunity. 2015;43(4):817-29.

2. Macia L, Thorburn AN, Binge LC, Marino E, Rogers KE, Maslowski KM, et al. Microbial influences on epithelial integrity and immune function as a basis for inflammatory diseases. Immunol Rev. 2012;245(1):164-76.

3. Smith PM, Howitt MR, Panikov N, Michaud M, Gallini CA, Bohlooly YM, et al. The microbial metabolites, short-chain fatty acids, regulate colonic Treg cell homeostasis. Science. 2013;341(6145):569-73.

4. Cummings JH, Pomare EW, Branch WJ, Naylor CP, Macfarlane GT. Short chain fatty acids in human large intestine, portal, hepatic and venous blood. Gut. 1987;28(10):1221-7.

5. Trompette A, Gollwitzer ES, Yadava K, Sichelstiel AK, Sprenger $\mathrm{N}$, Ngom-Bru C, et al. Gut microbiota metabolism of dietary fiber influences allergic airway disease and hematopoiesis. Nat Med. 2014;20(2):159-66.

6. Eeckhaut V, Machiels K, Perrier C, Romero C, Maes S, Flahou B, et al. Butyricicoccus pullicaecorum in inflammatory bowel disease. Gut. 2013;62(12):1745-52.

7. Machiels K, Joossens M, Sabino J, De Preter V, Arijs I, Eeckhaut $\mathrm{V}$, et al. A decrease of the butyrate-producing species Roseburia hominis and Faecalibacterium prausnitzii defines dysbiosis in patients with ulcerative colitis. Gut. 2014;63(8):1275-83.

8. Sommer F, Backhed F. The gut microbiota-masters of host development and physiology. Nat Rev Microbiol. 2013;11(4):227-38. 
9. Nicholson JK, Holmes E, Kinross J, Burcelin R, Gibson G, Jia W, et al. Host-gut microbiota metabolic interactions. Science. 2012;336(6086):1262-7.

10. Rooks MG, Garrett WS. Gut microbiota, metabolites and host immunity. Nat Rev Immunol. 2016;16(6):341-52.

11. Louis P, Hold GL, Flint HJ. The gut microbiota, bacterial metabolites and colorectal cancer. Nat Rev Microbiol. 2014;12(10):661-72.

12. Rothhammer V, Mascanfroni ID, Bunse L, Takenaka MC, Kenison JE, Mayo L, et al. Type I interferons and microbial metabolites of tryptophan modulate astrocyte activity and central nervous system inflammation via the aryl hydrocarbon receptor. Nat Med. 2016;22(6):586-97.

13. Ragsdale SW, Pierce E. Acetogenesis and the Wood-Ljungdahl pathway of $\mathrm{CO}(2)$ fixation. Biochim Biophys Acta. 2008;1784(12):1873-98.

14. Koh A, De Vadder F, Kovatcheva-Datchary P, Backhed F. From dietary fiber to host physiology: short-chain fatty acids as key bacterial metabolites. Cell. 2016;165(6):1332-45.

15. Gurav A, Sivaprakasam S, Bhutia YD, Boettger T, Singh N, Ganapathy V. Slc5a8, a Na ${ }^{+}$-coupled high-affinity transporter for short-chain fatty acids, is a conditional tumour suppressor in colon that protects against colitis and colon cancer under lowfibre dietary conditions. Biochem J. 2015;469(2):267-78.

16. Miyauchi S, Gopal E, Fei YJ, Ganapathy V. Functional identification of SLC5A8, a tumor suppressor down-regulated in colon cancer, as a $\mathrm{Na}(+)$-coupled transporter for short-chain fatty acids. J Biol Chem. 2004;279(14):13293-6.

17. Halestrap AP, Wang X, Poole RC, Jackson VN, Price NT. Lactate transport in heart in relation to myocardial ischemia. Am $\mathrm{J}$ Cardiol. 1997;80(3A):17A-25A.

18. Kim MH, Kang SG, Park JH, Yanagisawa M, Kim CH. Shortchain fatty acids activate GPR41 and GPR43 on intestinal epithelial cells to promote inflammatory responses in mice. Gastroenterology. 2013;145(2):396-406 (e1-10).

19. Macia L, Tan J, Vieira AT, Leach K, Stanley D, Luong S, et al. Metabolite-sensing receptors GPR43 and GPR109A facilitate dietary fibre-induced gut homeostasis through regulation of the inflammasome. Nat Commun. 2015;6:6734.

20. Fernandes J, Su W, Rahat-Rozenbloom S, Wolever TM, Comelli EM. Adiposity, gut microbiota and faecal short chain fatty acids are linked in adult humans. Nutr Diabetes. 2014;4:e121.

21. Vogt SL, Pena-Diaz J, Finlay BB. Chemical communication in the gut: effects of microbiota-generated metabolites on gastrointestinal bacterial pathogens. Anaerobe. 2015;34:106-15.

22. Canfora EE, Jocken JW, Blaak EE. Short-chain fatty acids in control of body weight and insulin sensitivity. Nat Rev Endocrinol. 2015;11(10):577-91.

23. Ruppin H, Bar-Meir S, Soergel KH, Wood CM, Schmitt MG Jr. Absorption of short-chain fatty acids by the colon. Gastroenterology. 1980;78(6):1500-7.

24. Bloemen JG, Venema K, van de Poll MC, Olde Damink SW, Buurman WA, Dejong $\mathrm{CH}$. Short chain fatty acids exchange across the gut and liver in humans measured at surgery. Clin Nutr. 2009;28(6):657-61.

25. Reichardt N, Duncan SH, Young P, Belenguer A, McWilliam Leitch C, Scott KP, et al. Phylogenetic distribution of three pathways for propionate production within the human gut microbiota. ISME J. 2014;8(6):1323-35.

26. Louis P, Young P, Holtrop G, Flint HJ. Diversity of human colonic butyrate-producing bacteria revealed by analysis of the butyryl-CoA: acetate CoA-transferase gene. Environ Microbiol. 2010;12(2):304-14.

27. Ze X, Duncan SH, Louis P, Flint HJ. Ruminococcus bromii is a keystone species for the degradation of resistant starch in the human colon. ISME J. 2012;6(8):1535-43.
28. Bhutia YD, Ganapathy V. Short, but smart: SCFAs train T cells in the gut to fight autoimmunity in the brain. Immunity. 2015;43(4):629-31.

29. Lin MY, de Zoete MR, van Putten JP, Strijbis K. Redirection of epithelial immune responses by short-chain fatty acids through inhibition of histone deacetylases. Front Immunol. 2015;6:554.

30. Vinolo MA, Rodrigues HG, Hatanaka E, Hebeda CB, Farsky SH, Curi R. Short-chain fatty acids stimulate the migration of neutrophils to inflammatory sites. Clin Sci (Lond). 2009;117(9):331-8.

31. Vinolo MA, Hatanaka E, Lambertucci RH, Newsholme P, Curi R. Effects of short chain fatty acids on effector mechanisms of neutrophils. Cell Biochem Funct. 2009;27(1):48-55.

32. Vinolo MA, Rodrigues HG, Hatanaka E, Sato FT, Sampaio SC, Curi R. Suppressive effect of short-chain fatty acids on production of proinflammatory mediators by neutrophils. J Nutr Biochem. 2011;22(9):849-55.

33. Nastasi C, Candela M, Bonefeld CM, Geisler C, Hansen M, Krejsgaard T, et al. The effect of short-chain fatty acids on human monocyte-derived dendritic cells. Sci Rep. 2015;5:16148.

34. Chang PV, Hao L, Offermanns S, Medzhitov R. The microbial metabolite butyrate regulates intestinal macrophage function via histone deacetylase inhibition. Proc Natl Acad Sci USA. 2014;111(6):2247-52.

35. Furusawa Y, Obata Y, Fukuda S, Endo TA, Nakato G, Takahashi $\mathrm{D}$, et al. Commensal microbe-derived butyrate induces the differentiation of colonic regulatory $\mathrm{T}$ cells. Nature. 2013;504(7480):446-50.

36. Haghikia A, Jorg S, Duscha A, Berg J, Manzel A, Waschbisch A, et al. Dietary fatty acids directly impact central nervous system autoimmunity via the small intestine. Immunity. 2016;44(4):951-3.

37. Park J, Kim M, Kang SG, Jannasch AH, Cooper B, Patterson J, et al. Short-chain fatty acids induce both effector and regulatory $\mathrm{T}$ cells by suppression of histone deacetylases and regulation of the mTOR-S6K pathway. Mucosal Immunol. 2015;8(1):80-93.

38. Ishikawa T, Nanjo F. Dietary cycloinulooligosaccharides enhance intestinal immunoglobulin A production in mice. Biosci Biotechnol Biochem. 2009;73(3):677-82.

39. Brown AJ, Goldsworthy SM, Barnes AA, Eilert MM, Tcheang L, Daniels D, et al. The orphan G protein-coupled receptors GPR41 and GPR43 are activated by propionate and other short chain carboxylic acids. J Biol Chem. 2003;278(13):11312-9.

40. Taggart AK, Kero J, Gan X, Cai TQ, Cheng K, Ippolito M, et al. (D)-beta-Hydroxybutyrate inhibits adipocyte lipolysis via the nicotinic acid receptor PUMA-G. J Biol Chem. 2005;280(29):26649-52.

41. Lukasova M, Malaval C, Gille A, Kero J, Offermanns S. Nicotinic acid inhibits progression of atherosclerosis in mice through its receptor GPR109A expressed by immune cells. J Clin Invest. 2011;121(3):1163-73.

42. Le Poul E, Loison C, Struyf S, Springael JY, Lannoy V, Decobecq ME, et al. Functional characterization of human receptors for short chain fatty acids and their role in polymorphonuclear cell activation. J Biol Chem. 2003;278(28):25481-9.

43. Maslowski KM, Vieira AT, Ng A, Kranich J, Sierro F, Yu D, et al. Regulation of inflammatory responses by gut microbiota and chemoattractant receptor GPR43. Nature. 2009;461(7268):1282-6.

44. Chen H, Assmann JC, Krenz A, Rahman M, Grimm M, Karsten $\mathrm{CM}$, et al. Hydroxycarboxylic acid receptor 2 mediates dimethyl fumarate's protective effect in EAE. J Clin Invest. 2014;124(5):2188-92.

45. Tang Y, Chen Y, Jiang H, Robbins GT, Nie D. G-protein-coupled receptor for short-chain fatty acids suppresses colon cancer. Int $\mathbf{J}$ Cancer. 2011;128(4):847-56. 
46. Sina C, Gavrilova O, Forster M, Till A, Derer S, Hildebrand F, et al. G protein-coupled receptor 43 is essential for neutrophil recruitment during intestinal inflammation. $\mathbf{J}$ Immunol. 2009;183(11):7514-22.

47. Vieira AT, Macia L, Galvao I, Martins FS, Canesso MC, Amaral FA, et al. A role for gut microbiota and the metabolite-sensing receptor GPR43 in a murine model of gout. Arthritis Rheumatol. 2015;67(6):1646-56.

48. Masui R, Sasaki M, Funaki Y, Ogasawara N, Mizuno M, Iida A, et al. $\mathrm{G}$ protein-coupled receptor 43 moderates gut inflammation through cytokine regulation from mononuclear cells. Inflamm Bowel Dis. 2013;19(13):2848-56.

49. De Filippo C, Cavalieri D, Di Paola M, Ramazzotti M, Poullet JB, Massart S, et al. Impact of diet in shaping gut microbiota revealed by a comparative study in children from Europe and rural Africa. Proc Natl Acad Sci USA. 2010;107(33):14691-6.

50. Agus A, Denizot J, Thevenot J, Martinez-Medina M, Massier S, Sauvanet $\mathrm{P}$, et al. Western diet induces a shift in microbiota composition enhancing susceptibility to adherent-nvasive $E$. coli infection and intestinal inflammation. Sci Rep. 2016;6:19032.

51. Vernia P, Gnaedinger A, Hauck W, Breuer RI. Organic anions and the diarrhea of inflammatory bowel disease. Dig Dis Sci. 1988;33(11):1353-8.

52. Singh N, Gurav A, Sivaprakasam S, Brady E, Padia R, Shi H, et al. Activation of Gpr109a, receptor for niacin and the commensal metabolite butyrate, suppresses colonic inflammation and carcinogenesis. Immunity. 2014;40(1):128-39.

53. Wlodarska M, Thaiss CA, Nowarski R, Henao-Mejia J, Zhang JP, Brown EM, et al. NLRP6 inflammasome orchestrates the colonic host-microbial interface by regulating goblet cell mucus secretion. Cell. 2014;156(5):1045-59.

54. Thorburn AN, Macia L, Mackay CR. Diet, metabolites, and "western-lifestyle" inflammatory diseases. Immunity. 2014;40(6):833-42.
55. Peng L, Li ZR, Green RS, Holzman IR, Lin J. Butyrate enhances the intestinal barrier by facilitating tight junction assembly via activation of AMP-activated protein kinase in Caco-2 cell monolayers. J Nutr. 2009;139(9):1619-25.

56. Tambuwala MM, Cummins EP, Lenihan CR, Kiss J, Stauch M, Scholz CC, et al. Loss of prolyl hydroxylase-1 protects against colitis through reduced epithelial cell apoptosis and increased barrier function. Gastroenterology. 2010;139(6):2093-101.

57. Karhausen J, Furuta GT, Tomaszewski JE, Johnson RS, Colgan SP, Haase VH. Epithelial hypoxia-inducible factor-1 is protective in murine experimental colitis. $\mathrm{J}$ Clin Invest. 2004;114(8):1098-106.

58. Kelly CJ, Zheng L, Campbell EL, Saeedi B, Scholz CC, Bayless $\mathrm{AJ}$, et al. Crosstalk between microbiota-derived short-chain fatty acids and intestinal epithelial HIF augments tissue barrier function. Cell Host Microbe. 2015;17(5):662-71.

59. Breuer RI, Soergel KH, Lashner BA, Christ ML, Hanauer SB, Vanagunas A, et al. Short chain fatty acid rectal irrigation for leftsided ulcerative colitis: a randomised, placebo controlled trial. Gut. 1997;40(4):485-91.

60. Scheppach W. Treatment of distal ulcerative colitis with shortchain fatty acid enemas. A placebo-controlled trial. GermanAustrian SCFA study group. Dig Dis Sci. 1996;41(11):2254-9.

61. Vernia P, Marcheggiano A, Caprilli R, Frieri G, Corrao G, Valpiani D, et al. Short-chain fatty acid topical treatment in distal ulcerative colitis. Aliment Pharmacol Ther. 1995;9(3):309-13.

62. Vernia P, Annese V, Bresci G, d'Albasio G, D'Inca R, Giaccari $\mathrm{S}$, et al. Topical butyrate improves efficacy of 5-ASA in refractory distal ulcerative colitis: results of a multicentre trial. Eur J Clin Invest. 2003;33(3):244-8.

63. Scheppach W, Sommer H, Kirchner T, Paganelli GM, Bartram P, Christl S, et al. Effect of butyrate enemas on the colonic mucosa in distal ulcerative colitis. Gastroenterology. 1992;103(1):51-6. 